\title{
Study of Magnetic Micro-Ellipses by Cantilever Sensor
}

\author{
K. SeČIAnska*, J. ŠoltÝs And V. CAMBel \\ Institute of Electrical Engineering, SAS, Dúbravská cesta 9, 84104 Bratislava, Slovakia
}

\begin{abstract}
In this paper, we propose a method for prototyping cantilever sensors by means of a modification of commercial atomic force microscopy cantilevers, using electron beam lithography and focused ion beam milling. To overcome obstacles with resist coating related to spin-coating of nonplanar 3D substrates, in this case of free-standing cantilevers, we propose a modified method based on spin-coating technique. An auxiliary atomic force microscopy chip was inserted below the cantilever to quasi-planarize the surface during spin-coating of electron beam resist. Magnetic micro-ellipses were prepared at the free-end of the cantilever by electron beam lithography. We propose a design of a cantilever sensor for the study of magnetic coupling between two cantilevers, prepared by focused ion beam milling. In ideal case, the coupling could be detected by a shift in resonance peaks. Attractive and repulsive forces between magnetic structures were shown by magnetic force microscopy.
\end{abstract}

DOI: 10.12693/APhysPolA.131.833

PACS/topics: 85.70.Ay, 85.85.+j, 81.16.Nd

\section{Introduction}

In the last decades, cantilever sensors have become well-known and wide-spread sensor platform, from physical and chemical sensors to biological disease diagnosis. In principle, any external perturbation can be detected by a deflection of the cantilever beam or by a shift in the cantilever's resonance frequency. Micro-cantilevers have gained popularity among conventional sensors because of their high sensitivity, selectivity, low cost, low power requirement, and a fast time response [1].

The requirement to investigate ever smaller magnetic objects at the submicron scale motivates the increasing need for high-sensitivity. Cantilever magnetometers have a faster time response and have proven to be suitable for measuring the magnetic moments of microand nanostructures. Moreover, a single electron spin was detected using the magnetic resonance force microscopy by custom-fabricated silicon cantilever with an attached magnetic tip [2].

Usually, cantilever-based sensors are based on the fabrication from planar silicon-on-insulator (SOI) substrates. Cantilevers are defined in a device layer by photolithography and chemically etched. In addition, the top of the device layer can be further patterned by electron beam lithography (EBL). However, there is an obstacle that the acid used to remove the buried oxide layer of a SOI wafer to release structures, also etches a variety of other materials, including metallic and magnetic thin films. The properties of such films could be influenced by etching process [3]. Therefore, it would be more convenient to release the cantilever first and then prepare the structures without damage caused by the acid. This approach is based on the fabrication of devices (cantilevers, resonators) and subsequently defining the micro/nanostructures. Common EBL involves an EB resist,

*corresponding author; e-mail: katarina.secianska@savba.sk which is usually applied by spin-coating. However, standard spin-coating technique fails to pattern non-planar surfaces and cannot cover pre-structured substrates with resist uniformly. A possible solution is offered by alternative methods of resist deposition, such as resist evaporation [4], spray coating [5] or ice $\left(\mathrm{H}_{2} \mathrm{O}\right)$ lithography [6]. Nevertheless, these are mostly laborious processes and require special equipment. Alternatively, resist deposition from a water surface (float coating) [7] is simple, but favorable only for optical resists and the results are not sufficiently reproducible for EB resists.

For the rapid prototyping of cantilever sensors we would like to overcome the time-consuming multistep cantilever fabrication, because for scientific purposes it is not necessary to batch fabricate large numbers of sensors. Instead, we used ready-made commercial atomic force microscopy (AFM) cantilevers and further modified them by EBL and focused ion beam (FIB). To overcome the mentioned obstacles during cantilever resist covering we designed a simple method based on spin-coating that can be employed for fabrication of any prototype cantileverbased sensors. To demonstrate the functionality of our method, we have fabricated a cantilever sensor for the study of magnetic microstructures.

\section{Sensor design and its characterization by magnetic force microscopy}

We propose a cantilever sensor for studying the switching field of the magnetic microstructures. Our idea is a sensor consisting of two cantilevers; each one of them with different resonance frequency. Magnetic microstructures are patterned on both cantilevers, enabling magnetic coupling of the cantilevers in external magnetic field. The coupling will be detected by a shift in the resonance frequencies of the sensor. In ideal case, the cantilevers coupled by attractive forces between magnetic microstructures would result in a single resonance peak in the sensors spectrum.

Ferromagnetic structures of elliptical shape (referred to as ellipses) are suitable candidates for this experiment. The magnetic structure of ellipses can be easily 
tuned by their geometry. Depending on the aspect ratio of an ellipse, a single domain magnetic state or a vortex state can be formed. Furthermore, the magnetization and switching field can be set by ellipse dimensions.

Here, we prepared the cantilever sensor by modification of a conventional AFM cantilever. In order to prepare two cantilevers, the AFM cantilever was cut into two longitudinal parts. An array of 13 ellipses was fabricated on each of them. The aspect ratio of the ellipses was convenient for the single domain magnetic state. The size of the ellipses on each part was different, allowing to switch their magnetization separately, as higher external magnetic field is needed to switch the magnetization of smaller ellipses.

We inspected functionality of the proposed sensor by magnetic force microscopy (MFM). It is difficult to perform MFM scans of ellipses placed on the free-standing cantilevers. Therefore, we prepared ellipses of identical shape on planar substrate by the same fabrication process as will be described in the next paragraph for cantilever sensor. MFM scans in Fig. 1. show behavior of ellipses after magnetizing by external field, as well as illustrate three basic states of proposed cantilever sensor. Dark and light areas correspond to positive and negative poles of magnetic dipoles. Ellipses were in single domain magnetic states as expected.
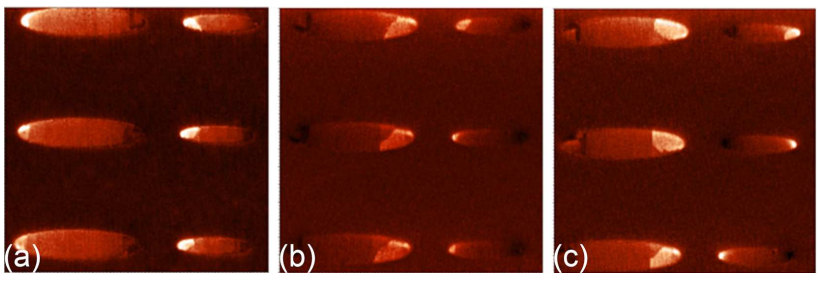

Fig. 1. MFM of magnetic ellipses. Remanent state, after ellipses were magnetized in magnetic field of: (a) $+100 \mathrm{mT}$, (b) $-5 \mathrm{mT}$, (c) $-6 \mathrm{mT}$. Size of the scans is $15 \times 15 \mu \mathrm{m}^{2}$.

By applying a sufficient in-plane external magnetic field along the longer axes of the ellipses, single domain state was introduced in both arrays of ellipses with identical orientation of the magnetization, as shown in Fig. 1a. In this case, cantilevers would be magnetically coupled by attractive forces between ellipses and they would respond at the same frequency. The frequency spectrum of the sensor would be characterized by a single resonance peak.

By gradual application of opposite magnetic field, at certain value the dipoles of larger ellipses switched into opposite direction. Figure 1b corresponds to repulsive forces between arrays of ellipses. In this case, the frequency spectrum of the sensor would be characterized by two separate resonance peaks corresponding to each cantilever.

The last studied case, the intermediate state, is shown in Fig. 1c. This case occurs because real magnetic ellipses are not exactly of the same shape (edge roughness); resulting in slightly different switching field. The frequency spectrum would be characterized by broadening of single peak, or previous separate peaks shift their positions into new values.

In other words, the different character of resonance peaks could be possibly utilized for a magnetic sensor to study magnetic properties of the prepared ellipses (or arbitrary magnetic structures in general). For example, mutual orientation of magnetic dipoles can be noninvasively inspected, whether dipoles are oriented in the same or in the opposite directions.

\section{Resist coating on cantilever}

The fabrication process started with two identical commercial silicon AFM chips. First, they were cleaned in oxygen plasma for 3 minutes. One of them was glued on a flat substrate. We used sapphire sample holder with a spring. A drop of polymethyl-methacrylate (PMMA) was used as a gluing agent and it was dried on a hot plate at $170{ }^{\circ} \mathrm{C}$ for $1 \mathrm{~min}$. The other chip had its cantilever removed and it was inserted below the cantilever of the glued chip. This step is schematically depicted in Fig. 2a. The procedure was done using a micromanipulator. The chip was finally fixed with a spring to the substrate. The substrate with chips in this configuration was loaded on the spin-coater. Several drops of PMMA $950 \mathrm{~K}$ resist were spin-coated for $45 \mathrm{~s}$ at $5000 \mathrm{rpm}$ onto the chip's surface. The resist was baked on a hot plate at $170^{\circ} \mathrm{C}$ for $2 \mathrm{~min}$. Chips uniformly coated with resist film can be seen in Fig. $2 \mathrm{~b}$.

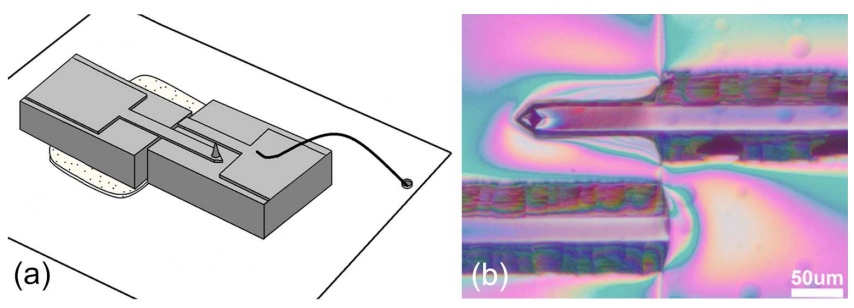

Fig. 2. (a) Illustrational scheme of the AFM chips glued on the flat substrate. (b) Chips and the cantilever uniformly coated with PMMA resist by spin-coating.

\section{Fabrication of cantilever sensor by EBL}

EBL on the cantilever (Fig. 2b) was performed using Raith EBL system with acceleration voltage of $30 \mathrm{kV}$ and exposure dose $300 \mu \mathrm{C} / \mathrm{cm}^{2}$. The exposed pattern was a design of 13 pairs of shorter and longer ellipses organized in two arrays. The sizes were $4 \mu \mathrm{m} \times 0.8 \mu \mathrm{m}$ and $7 \mu \mathrm{m} \times 1.5 \mu \mathrm{m}$, respectively. To reduce magnetic interaction between the neighboring ellipses within one array, the center-to-center distance was $6.5 \mu \mathrm{m}$. We prepared the higher number of ellipses to enhance mutual magnetic forces between the arrays. The distance between arrays was $2.5 \mu \mathrm{m}$. The resist was developed for $1 \mathrm{~min}$ in a 1:3 solution of methyl-isobutyl-ketone (MIBK) and isopropyl alcohol (IPA). The cantilever with exposed and developed resist can be seen in Fig. 3. The thickness of developed resist was measured by AFM. It was in the range of 70-100 nm which was sufficient to perform liftoff properly. 


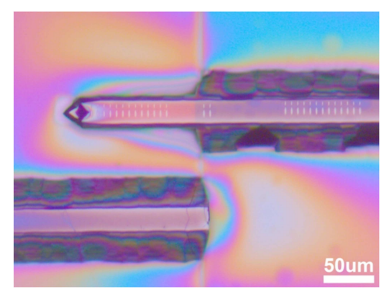

Fig. 3. The cantilever after EBL and development of the resist.
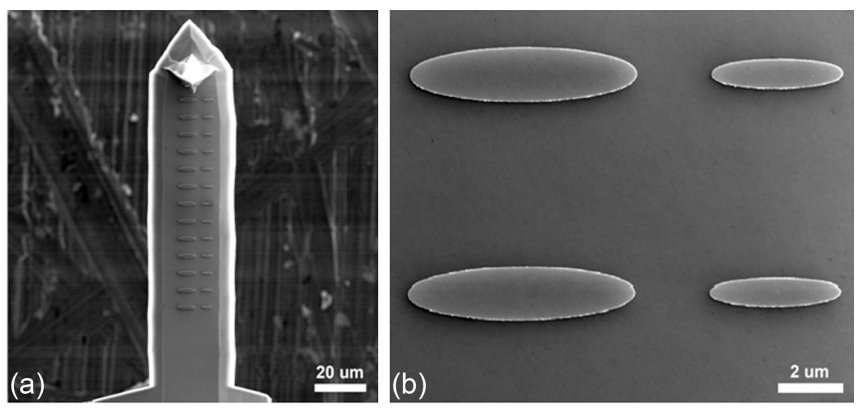

Fig. 4. Top-view SEM micrographs of (a) the cantilever after deposition of Py and lift-off, (b) detail of patterned ellipses.

Next, the $40 \mathrm{~nm}$ thin film of permalloy $\mathrm{Ni}_{80} \mathrm{Fe}_{20}$ was deposited by evaporation. Afterwards, resist lift-off was carried out in acetone for about half an hour. The chips were held together only by the resist layer, so they detached from each other during this step. At last, few seconds of ultrasonic agitation was necessary for complete lift-off. The cantilever after lift-off can be seen in Fig. 4.

Finally, the prepared cantilever was cut by FIB milling. The sharp tip at the end of the cantilever was cut off and the cantilever was cut into two longitudinal parts in between the ellipses arrays. The milling process employed $\mathrm{Ga}^{+}$column accelerated at $30 \mathrm{kV}$ and the ion current was $0.3 \mathrm{nA}$. Figure $5 \mathrm{c}$ shows the final cantilever sensor design.

\section{Discussion}

The chip with cantilever sensor (Fig. 5c) was inserted in the AFM measuring head. The spot size of laser beam was large enough to focus on both parts. The frequency spectrum of the sensor was identified using AFM control software. Our aim was to observe changes in frequency spectrum depending on applied external magnetic field. We expected a change from two separate resonance peaks into one resonance peak caused by magnetic coupling between cantilever parts (i.e. ellipses). We applied in-plane homogeneous magnetic field along the long axes of ellipses with values ranging from $100 \mathrm{mT}$ to $-100 \mathrm{mT}$. Even though we tried to initiate the coupling and the states visible in Fig. 1, we did not observe any shift. We assume our sensor sensitivity was probably not sufficient. For the future, we are working on improvement of the
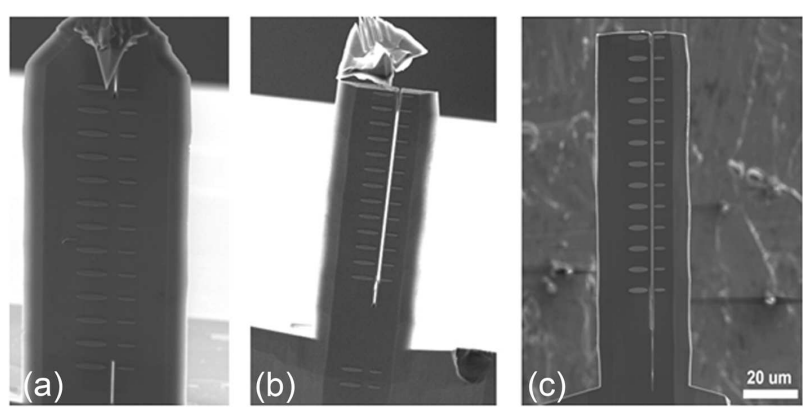

Fig. 5. The procedure of cutting the cantilever into parts: (a) definition of marks, (b) procedure of removing the tip, (c) final cantilever sensor design.

sensitivity of the proposed sensor. First, the cantilever was probably too thick $(\approx 2 \mu \mathrm{m})$ hence it had high spring constant $(\approx 16 \mathrm{~N} / \mathrm{m})$. Reduction of a cantilever thickness will lead to increase sensitivity. Second, distance between magnetic structures was too large $(2.5 \mu \mathrm{m})$ and mutual forces were too low. Minimalization of distance will increase magnetic coupling. Our aim is to prepare magnetic structures located less than $1 \mu \mathrm{m}$ from each.

\section{Conclusion}

To conclude, in this work we proposed a magnetic cantilever sensor for study of magnetic structures. We fabricated magnetic microstructures on a commercial AFM cantilever by modified spin-coating process and EBL. We showed the principle of functionality of proposed sensor by performing series of MFM measurement in the external magnetic field. Moreover, we fabricated the final cantilever sensor, but the results were negative presumably due to a high spring constant of the cantilevers and a large distance between the arrays. Our future plans are to increase the sensitivity of the sensor.

\section{Acknowledgments}

This work was supported by Structural Funds of the European Union by means of the Research Agency of the Ministry of Education, Science, Research and Sport of the Slovak republic in the project "CENTE I" ITMS code 26240120011 and by VEGA project 2/0183/15.

\section{References}

[1] A. Boisen, S. Dohn, S.S. Keller, S. Schmid, M. Tenje, Rep. Prog. Phys. 74, No. 3 (2011).

[2] D. Rugar, R. Budakian, H.J. Mamin, B.W. Chui, Nature 430, 6997 (2004).

[3] J. Losby, J.A.J. Burgess, C.M.B. Holt, J.N. Westwood, D. Mitlin, J. Appl. Phys. 108, 12391 (2010).

[4] J. Zhang, C. Con, B. Cui, ACS Nano 8, 3483 (2014).

[5] J. Linden, Ch. Thanner, B. Schaaf, S. Wolff, B. Lägel, E. Oesterschulze, Microelectron. Eng. 88, 2030 (2011).

[6] A. Han, A. Kuan, J. Golovchenko, D. Branton, Nano Lett. 12, 1018 (2012).

[7] H. Zhou, B.K. Chong, P. Stopford, G. Mills, A. Midha, J. Vac. Sci. Technol. B 18, 3594 (2000). 